\title{
Italique
}

Poésie italienne de la Renaissance

XVII | 2014

Varia

\section{Sulle «amorose tenerezze» del Marino. Tra Epitalami ed Adone}

Emilio Russo

\section{OpenEdition}

Édition électronique

URL : http://journals.openedition.org/italique/390

DOI : 10.4000/italique.390

ISSN : 1663-4438

Éditeur

Librairie Droz

Édition imprimée

Date de publication : 1 octobre 2014

Pagination : 141-162

ISBN : 978-2-600-01841-8

ISSN : 1423-3983

\section{Référence électronique}

Emilio Russo, « Sulle «amorose tenerezze» del Marino. Tra Epitalami ed Adone », Italique [En ligne], XVII I 2014, mis en ligne le 01 octobre 2016, consulté le 10 décembre 2020. URL : http://

journals.openedition.org/italique/390; DOI : https://doi.org/10.4000/italique.390

(c) Tous droits réservés 
Emilio Russo

S ULLE «A M OR OSE TENEREZZE»

D E L M A R I N O.

TRA EPITALAMI ED ADONE 



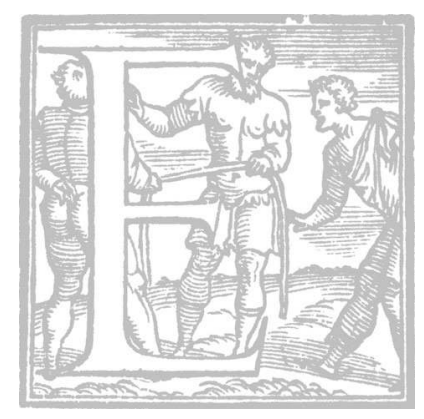

ntro gli esordi del Marino, tra la Napoli degli ultimi due decenni del Cinquecento e i primi anni romani, la pratica di una poesia tra sensuale e lasciva rivestì il ruolo di un crinale privilegiato. Accanto all'encomiastica obbligata, e accanto alle prime celebrazioni ammirate di opere d'arte ospitate in gallerie illustri, e anzi tutto nella collezione di Matteo di Capua, esercizi poetici di marca lasciva scandiscono gli esordi del Marino, con la vetta rappresentata dalla canzone dei baci, diffusasi rapidamente per via manoscritta, e primo reale biglietto di presentazione al di fuori dell'ambiente napoletano. ${ }^{\mathrm{I}}$ Mentre la dorsale dei capitoli burleschi, di matrice bernesca, dal Camerone allo Stivale, rispondeva ad altre istanze, ${ }^{2}$ sonetti madrigali e canzoni si spingevano in alcune occasioni alla ricerca di un equilibrio tra la raffinata tessitura ritmica, la riscrittura accorta dei precedenti letterari e un tenue velo di metafora a coprire una materia spesso assai esplicita. ${ }^{3}$

Era un terreno battuto in modo strumentale, alla ricerca di una affermazione vistosa, e non solo dal Marino: si ricordi, per citare un percorso destinato a correre a lungo parallelo, l'esordio di Stigliani con le Rime del I60I e poi del I605, rime gravide di Enigmi osceni e puntualmente sanzionate dal Sant'Uffizio in una prima tornata censoria sulla lirica contemporanea. ${ }^{4}$ Proprio in virtù di quel contesto di riferimento, e del rischio dunque di interventi da parte delle congregazioni cardinalizie, questa pratica poetica assumeva una valenza specifica, valeva cioè esperire un rischio calcolato e un terreno di confine, con oscillazioni che andavano tra la circolazione manoscritta dei testi e una declinazione pubblica e appena cifrata della materia oscena. ${ }^{5}$ E che il Marino avesse individuato in tutta consapevolezza le dinamiche, scegliendo ora l'una ora l'altra soluzione, lo dice uno scorcio del Ragguaglio a Carlo Emanuele, uno dei primi passaggi difensivi che fu costretto a congegnare in una fase delicata, nelle prime settimane del I609, le settimane calde dell'affaire Murtola:

Non niego io, che per accomodarmi all'umor del secolo, per lusingare l'appetito del mondo, e per rendere lo stile vezzoso morbido et attrattivo, non mi sia dilettato alquanto delle amorose tenerezze, e che non si possa 
dalle mie cose raccogliere alcun cenno di metafora, la qual con giocosa allegoria alluda a qualche lascivo sentimento, appena però penetrabile a gl'intelletti svegliati et arguti. Niego bene ch'io abia giamai smascheratamente in esse trattato di cose sozze, onde a ragione mi possa Platone discacciar dalla sua Republica come pernicioso a' costumi e corrompitore della gioventù. Se io per sì debole querela debbo esser condannato, perché non si condanna tutta quanta la Poesia, la qual cotali licenze porta seco? Quanti meno onesti e più disvelati concetti si ritrovano sparsi in libri d'antichi e di moderni scrittori, i quali tuttavia e s'imprimono, e si vendono, e si leggono, e si permettono? ${ }^{6}$

Pagina certo funzionale, priva di caratura teorica, e tuttavia nella quale si segnava un confine generalissimo tra una cifra più o meno leggibile del testo, e l'offerta senza veli della materia oscena ("cose sozze»); pagina in ogni caso rivelatrice di una inclinazione specifica, con lo "stile morbido e attrattivo", le allusioni svolte attraverso una "giocosa allegoria", mai dunque in modo piano e dichiarato, e il supporto di una ininterrotta tradizione letteraria, dai greci ai contemporanei. Accenti prossimi a quelli incastonati al principio del canto VIII dell'Adone, il canto intestato ai Trastulli amorosi e culminante nell'amplesso dei due amanti, ${ }^{7}$ nel quale era difesa la «molle e lusinghiera Musa» che dettava «poesie si tenere e lascive» (VIII 3), sulla base della difesa dettata da Marziale («che s'oscena è la penna, è casto il core» VIII ठ). E non è difficile intravedere in queste formule la rete di argomentazioni che doveva garantire sul proscenio delle corti o delle accademie, se non nel registro ufficiale delle stampe, una poesia intrisa di «lascivo sentimento».

Su questo terreno, e lasciando per ora da parte la questione del poema maggiore, Marino si mostrò disposto non solo a rimanere, ma anche a difese tenaci e persino rischiose. È quanto emerge ad esempio dalle dinamiche relative alla stampa di Lira III nel I6I4, sia per la presenza nella zona conclusiva dell'edizione stampata da Ciotti delle ottave dichiaratamente erotiche del Duello amoroso, ${ }^{8}$ sia per l'interessamento richiesto dal Marino a Girolamo Magagnati affinché proteggesse dai tagli censori le zone più azzardate della raccolta. ${ }^{9}$ Accanto al Duello amoroso, nel corso degli anni, vanno almeno ricordati $i$ Trastulli estivi e gli Amori notturni, prove di una poesia ripresa e garantita da Ovidio" Io appunto distinta dalle caricature "smascheratamente" oscene che il Marino riservava, sia pure con il diaframma della solita prudenza, alla circolazione riservata ma non ristrettissima 
di amici e sodali. Cosi, ad esempio, nel sonetto $\mathrm{O}$ belle agl'occhi miei chiappe latine, riscrittura parodica e in chiave omoerotica di uno dei luoghi più celebri e ammirati della Liberata (VI 99: O belle agli occhi miei tende latine), testo che si legge assegnato al Marino nel Vat. Lat. 9226, e poi in alcuni altri manoscritti, talora con attacco persino più crudo. "' Senza apprezzabili soluzioni di continuità, dunque, si definisce una linea che, fondata sullo scrivere «tenero e lascivo», passava dalla sequenza di settenari ribattuti della canzone dei baci alla riscritture parodiche e grevi o ancora agli attacchi polemici più virulenti, a costituire un insieme importante nell'affermazione mariniana del primo quindicennio del secolo.

Un contesto, quello qui rapidamente tracciato, fattosi più dettagliato grazie alle indagini negli ultimi anni, e dal quale occorre muovere per una valutazione su terreni contigui, ancora a matrice lirica e tuttavia caratterizzati da un gradiente diverso di ufficialità. Significativo è infatti che la ricerca di un equilibrio azzardato venisse dal Marino attuata anche in occasione di diversi tra gli Epitalami, in prove di omaggio e di celebrazione ufficiale che escludevano per statuto, sin dal consueto esito a stampa, la fruizione in penombra. Celebrando le principesse di Savoia o una figura del calibro di Giovan Carlo Doria, o ancora Ercole Pepoli ed Elena Aldobrandini, congiunta di Clemente VIII, Marino spingeva allo stesso modo $i$ suoi versi fino ad esiti di aperta lascivia, descrivendo compiaciuto l'amplesso degli sposi, sceneggiandone passaggi e tappe fino al culmine del piacere. Era una pratica almeno in parte legittimata dalla tradizione classica, da Catullo a Claudiano, ${ }^{\mathrm{I} 2}$ e che Marino mirava a rinnovare, ${ }^{\mathrm{I}}$ alternandola ad altre prove epitalamiche più composte, ove il corredo mitologico si dispiegava in scene e movenze aggraziate. Rispondendo a questa varietà tonale, la collezione degli Epitalami si sedimentò negli anni, e nella Lettera Claretti del I6I4 Marino elencava cosi i testi:

Vi sono oltra ciò Epitalami, alcuni in canzoni altri in verso sciolto, cioè Urania, Imeneo, Amore, Ercole, Le Muse, Il Sogno, L'Annello, Il Torneo, Il Ballo, La Cena, Il Letto, fatti tutti in occasione di nozze di diversi Prencipi e Signori. ${ }^{14}$

Rispetto a questo diagramma la stampa del I6I6, licenziata a Parigi $e$ con in apertura la Francia consolata per le nozze di Luigi $X I I I,{ }^{\mathrm{Is}}$ presentava alcune variazioni: cadevano nella sequenza i titoli 
Amore Muse e Sogno, annunciati solo un paio d'anni prima e sui quali mancano notizie ulteriori, e veniva invece aggiunta Venere pronuba, oltre appunto alla Francia consolata. E se la sistemazione della princeps parigina scompaginava la sequenza cronologica dei testi, rispondendo a un principio di variatio che doveva sottolineare la ricchezza della raccolta, ${ }^{16}$ nell'insieme almeno quattro, sia pure con diversa insistenza, erano le tessere che si spingevano su un versante lascivo: la Venere pronuba, appunto, Il letto, Le fatiche d'Ercole $e$ Imeneo.

Con riferimento a questi testi converrà però qui prescindere dalla loro sede nella princeps e riferirsi ai tempi di composizione, con tutta verosimiglianza agganciati alla cronologia delle nozze celebrate, a partire dunque da quell'Imeneo dedicato ad Antonio Carafa ed Elena Aldobrandini già nel I602 da un Marino appena entrato nell'orbita degli Aldobrandini. ${ }^{17}$ Articolato nello schema metrico abCabC cDdeeFF, l'epitalamio lascia sullo sfondo in apertura gli amori di Aminta ed Amarilli, ${ }^{18}$ e si offre come una registrazione della voce di Fileno, una voce le cui modulazioni rusticamente Tirsi incide sulla corteccia dei boschi. ${ }^{19}$ Il canto si apre con un'invocazione alla Notte, ricca sul piano della ripresa della tradizione letteraria, ${ }^{20}$ e sulla preghiera al carro del Sole di affrettare il corso lasciando luogo alle tenebre propizie agli amori. Su questa cornice si innestano versi su Amore come divinità innocente, compagno necessario di Imeneo:

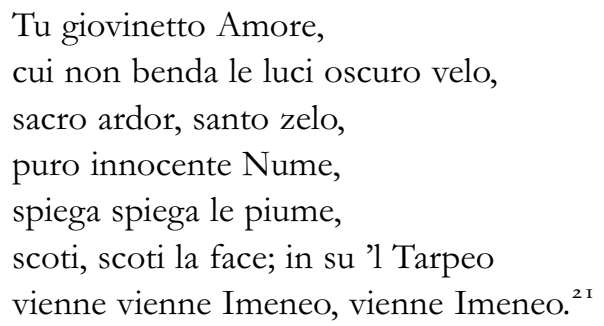

Accenti nel quali pare intravedersi la commistione sacro/profano che sarebbe poi divenuta elemento nevralgico nelle opere del Marino e che qui prelude alla descrizione della fanciulla ritrosa, bella e selvaggia, cui è rivolto il topico invito a godere dei piacere amorosi, a scacciare un «rossore intempestivo» lasciando corso ad altro contegno: 


\section{Castamente lascivo}

Tinga un pallor soave il vago aspetto,

ceda il foco del volto a quel del petto. ${ }^{22}$

L'equilibrio precario, ambiguo, tra castità e lascivia si rivela nei versi successivi non più che l'accostamento tra una pudicizia esibita, iniziale, e una passione sottostante, mordace, che il giovane "Garzon» doveva cogliere con audacia:

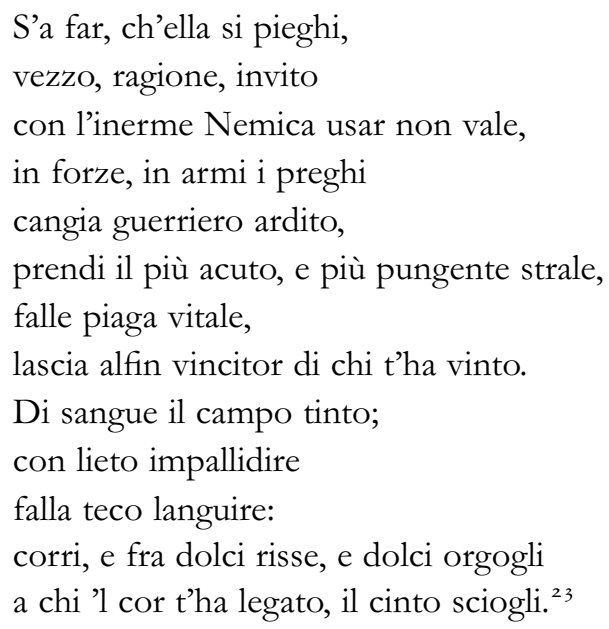

A una serie di elementi arditi ma previsti dal codice - lo sciogliersi del cinto verginale, l'accento sul sangue della sposa $-{ }^{24}$ Marino aggiunge il doppio senso dello strale, giustificato nell'orizzonte della guerra amorosa tra la Nemica e il guerriero ardito, e tuttavia con un elemento di cruda e fisica oscenità, che attribuisce tono specifico all'omaggio e anticipa certi passaggi in rilievo dell'Adone. ${ }^{25}$ Dopo quest'acme la notte si quieta nelle sue ore più profonde, e l'unione degli sposi è finalmente benedetta da Imeneo, con annuncio del frutto di un erede al solito destinato ad alte imprese. Cosi si chiude il canto di Fileno registrato da Tirsi: "Tacque ciò detto, ed ambo / felici amanti tra felici amori / Amor gli strinse, $e$ strinse $i$ corpi e $i$ corì, ${ }^{26}$ con un passaggio che ricorda da vicino Adone, VIII 89 ( $«$ Amor gli stringe e stringe $i$ corpi e $i$ cori / con lacci indissolubili e tenaci»), ma che coincide quasi con l'incipit di uno dei sonetti epitalamici compresi nella raccolta del I6I6 e già composto a inizio secolo per Margherita Aldobrandini e Ranuccio Farnese (Stringe Imeneo duo corpi, e i corpi e i cori). ${ }^{27}$ 
Primi segni di una circolarità di presenze e di riferimenti: il legame degli Epitalami con l'Adone, tanto in termini di sovrapposizioni puntuali, quanto e soprattutto in termini di una poetica omologa nel trattamento della materia lasciva, può rappresentare una chiave di lettura significativa. Si deve a Michele Dell'Ambrogio, ad esempio, sulla scorta di una indicazione al solito illuminante presente nel commento mondadoriano di Giovanni Pozzi, ${ }^{28}$ l'aver sottolineato il rapporto del canto VIII del poema, intestato ai Trastulli, certo uno dei punti piu avanzati della sperimentazione sensuale del Marino, e Il letto, il sorprendente testo licenziato per le nozze tra Francesco Gonzaga e Margherita di Savoia nella primavera del 1608. ${ }^{29}$ A differenza de Il balletto delle Muse, composto in parallelo per le nozze tra Alfonso III d'Este e Isabella di Savoia, ${ }^{30}$ Il letto, articolato in strofe abbAacCdD, sceneggiava una nuova versione del duello amoroso tra $i$ due illustri coningi. L'attacco riprendeva la descrizione della notte conveniente agli amori ma la ambientava all'interno di un «real soggiorno», ambientazione sontuosa conveniente allo status principesco degli omaggiati, che erano cosi presentati:

Inquieti riposi

tra molli lini e bianchi

traean languidi e stanchi

Margherita e Francesco, i regii sposi;

e 'n arringhi amorosi,

dove l'ire, e i contrasti

erano vezzi, ove vezzosi, e casti

erano scherzi gli schermi

guerreggiavano in pace, atleti inermi.

Fu lor campo e steccato

cameretta soave,

la cui secreta chiave

volgea fido custode Arciero alato.

Qui con l'Eroe bennato

era franca ed ardita

la leggiadra Aversaria a fronte uscita.

Eran loriche, e scudi

contro i teneri colpi i seni ignudi.

Gran padiglione in alto

facea raccolto in giro 


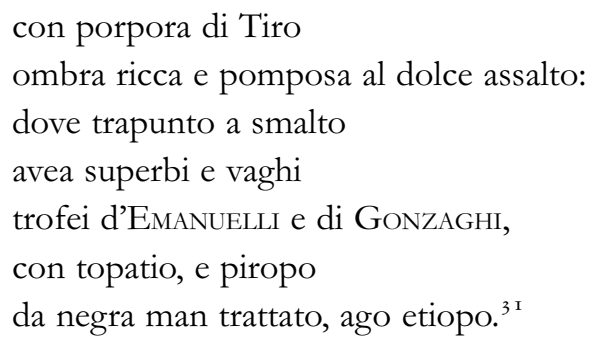

Anche in questo scorcio risultano evidenti $i$ punti di contatto con la sezione dell'Adone dedicata all'amplesso dei due amanti, dalla sontuosa cornice che li ospita (la cameretta di VIII 9I, la descrizione del talamo prezioso delle ottave seguenti) fino all'estasi e all'abbandono della fine dello stesso canto VIII. Entro $i$ versi de Il letto, poi, è significativa nella sua ambiguità l'aggiunta di quel singolare rispecchiamento per cui gli eroi delle due casate, effigiati da mano abilissima d'artefice sul padiglione mentre compiono le azioni più gloriose, osservano dall'alto le volute della lotta amorosa. Un gioco di specchi interno al codice della celebrazione, e tuttavia carico di quella linea dissacrante, profondamente antiepica, che percorre la poesia mariniana. Proprio la collocazione delle nobilissime conquiste quale sontuosa cornice dell' amplesso distribuisce in silenzio gli ordini relativi, rivela le gerarchie assunte dalla celebrazione mariniana, qui come altrove orientata a rispettare solo parzialmente i moduli propri dell'encomastica. Sotto quei precedenti gloriosi $i$ due amanti conducevano «risse d'amor pudiche», alternando tra loro «dolci disfide», fino al diluvio di baci:

Pioveano i baci a groppi,

grandinavano a mille.

Quante il foco ha faville

atomi il sol, cotanti erano gli scoppi.

Amor tenaci e doppi

più che d'edre o di polpi

ordiva i nodi, e raddoppiando i colpi

de' baci senza fine

il numero scrivea su le cortine. ${ }^{32}$

La fenomenologia del bacio è stata a lungo indagata e sono noti gli esiti di virtuosismo raggiunti dal Marino nella già ricordata canzone dei baci, avantesto di quella rielaborazione che si legge in Adone, 
VIII I24-4I. ${ }^{33}$ Dopo la breve pausa di uno sguardo innamorato e di una preghiera accorata dello sposo, dopo che il corteggio degli amorini ha approntato il talamo, il nuovo amplesso si chiude nel giro di pochi versi. ${ }^{34} \dot{E}$ il velo stesso di Amore, con un altro particolare crudo e di viva valenza simbolica, ${ }^{35}$ a cogliere il sangue virginale ("de la prima ferita il primo sangue»), ma è soprattutto il culmine del piacere degli amanti, descritto da Marino nei versi subito precedenti, ad offrire un altro passaggio in rilievo:

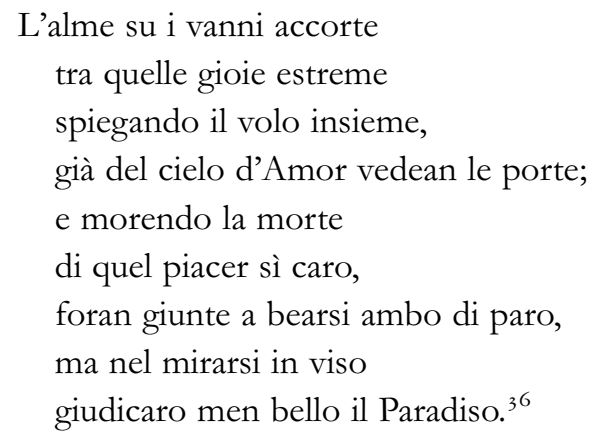

Rapite nel cielo d'Amore (vd. anche Adone VIII I48) grazie ai piaceri supremi dell'unione sensuale, le due anime sfiorano una beatitudine divina e tuttavia preferiscono, con uno scarto che doveva quanto meno risultare ambiguo, la dolcezza tutta terrena del piacere. Declinando dunque il registro peculiare di un omaggio ufficiale, Marino innestava in alcuni degli epitalami tratti di una poetica nitida sia negli indirizzi ideologici e nella tessitura stilistica. La rielaborazione dei motivi classici era condotta in un dettato rapido, scandito sui settenari, punteggiato di bisticci e allitterazioni, organizzato su un intrico di ossimori e duplicazioni, con tratti di quel panneggio che poi andava a connotare in modo più ampio le prove schiettamente mitologiche (si pensi a Urania). Al contempo, malgrado la franchigia concessa al patrimonio legato a Imeneo, Marino spingeva la celebrazione dell'amore sensuale fino al punto di ridimensionare sottilmente, e di incrinare, ideali sovraordinati, a partire da quello cardine della virtù.

Cosi anche ne Le fatiche d'Ercole, testo probabilmente composto nel I609 (metro aaBaC), nel quale il rilievo meno marcato degli omaggiati e la loro stessa onomastica - il conte Ercole Pepoli e Vittoria Cibo - ${ }^{37}$ indirizzavano a una più leggera e divertita rielaborazione delle imprese 
di Ercole sotto la formula di vittorie amorose, con declinazioni ora liriche e sentimentali, ora più audaci:

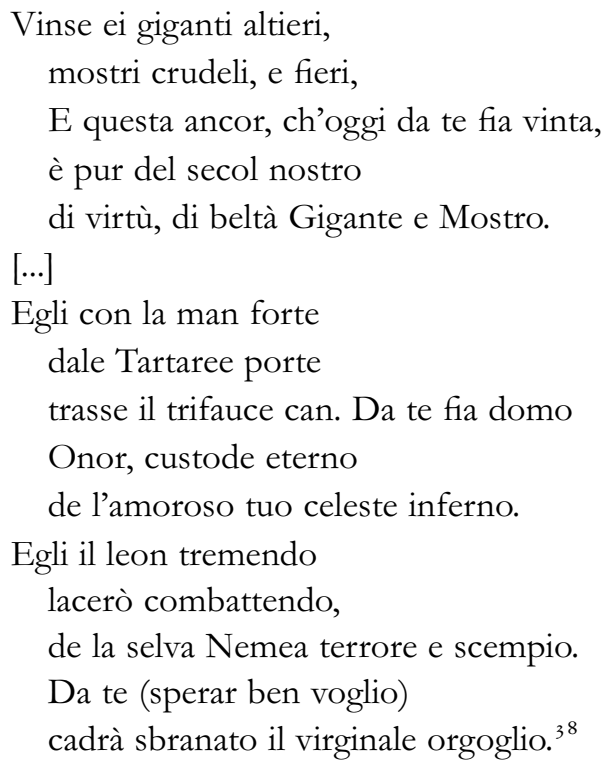

Un modulo di rielaborazione particolarmente congeniale alla poesia mariniana, secondo un rispecchiamento tra due piani che venivano congiunti a ogni tornata per via ingegnosa; e su questo modulo, su questa stessa tessitura metrica Marino aveva imperniato sin dai prima anni del secolo il progetto di una raccolta di canzonette, la Polinnia. ${ }^{39}$ In questo caso gli accenti sensuali erano limitati a punte isolate, soprattutto nella chiusura concettosa della singola strofe, e in genere riassorbiti nel fluire rapido degli anelli poetici, che aveva alle spalle anche la rielaborazione del Claudiano di In Rufinum, III 283 sgg. e di uno scorcio del De raptu Proserpinae. ${ }^{40}$ Esiti assai diversi, per tornare a un precedente certamente noto al Marino, aveva sortito una parallela prova tassiana, un componimento a celebrazione delle nozze di Ercole Tasso, giocato in misura assai più discreta sul precedente mitologico di Alcide (Tasso, Rime, I2sI).

É comunque nell'impianto più articolato e impegnativo della Venere pronuba che le scelte mariniane risultano marcate. Individuato anche nella penultima stagione come testo esemplare della maniera licenziosa del Marino, Venere pronuba è stato più volte oggetto di indagine per la libertà e l'azzardo cui Marino spinse la celebrazione 
delle nozze di Giovan Carlo Doria e Veronica Spinola. E si deve forse proprio alla natura del testo la scelta di Marino di tacerne la disponibilità all'altezza del I6I4 (l'epitalamio era stato composto nel I608), all'altezza della Lettera Claretti, ${ }^{4 \mathrm{I}}$ e quella complementare e di segno opposto di collocare l'omaggio al Doria in bella mostra, in terza sede, nella princeps del I6I6, entro il contesto assai meno rigido della corte francese. Va peraltro notato che anche le stampe italiane degli Epitalami si susseguirono senza scosse, e che solo nel 1678 , in quella che può essere considerata una tarda recrudescenza, Venere pronuba venne ufficialmente iscritto all'Indice, giusto accanto a quel Duello amoroso che aveva conosciuto circolazione intermittente sin dal I6I.4. ${ }^{42}$ Fu probabilmente il rapporto con Giovan Carlo Doria, cementato intorno a una comune passione figurativa, ${ }^{43}$ oltre che la scelta di riprendere da vicino il modello di Claudiano, ${ }^{44}$ a indirizzare la scrittura mariniana, dopo una prima sezione più narrativa e misurata, verso una lezione impartita da Venere al giovane sposo, una lezione sull'arte dell'amplesso; e la figura della dea in questo caso pare svolgere la funzione di un tenue velo letterario, sovrapposto a una comunicazione divertita tra sodali. Il punto di svolta, poco segnalato in bibliografia, era rappresentato dalla celebrazione degli imenei da parte della stessa Venere, secondo però un ideale di voluttà che assegnava tinte ambigue, se non trasgressive, al sacramento:

E Citerea

il cui virile aspetto

facea feroce e franco

del gran valor de' suoi fede assai chiara.

Con tenace legame indi de' duo

congiunte ambe le destre,

pronuba e sposatrice

gli imenei celebrò con questi accenti.

'Vivete omai concordi,

e le nostre dolcezze

imparate a godere.

Suonino mille baci

di nettare umidetti'. ${ }^{45}$ 
Da qui, da questo taglio edonistico, procedeva la prefigurazione dell'unione sessuale, sempre presentata nella forma di una dolce contesa amorosa, tra le lagrime iniziali della sposa e il montare progressivo del piacere. Appunto in relazione a Venere pronuba Angelo Borzelli, in pagine ormai lontane, proponeva l'ipotesi che l'elemento della voluttà si sviluppasse nei versi mariniani a partire dagli ideali di un naturalismo eterodosso proposti in quegli anni da Giulio Cesare Vanini, e andasse dunque letto come una testimonianza, implicita quanto proterva, di una prospettiva ideologica poi piu ampiamente dispiegata nell'Adone. ${ }^{46}$ I rapporti tra Marino e Vanini, dall'amicizia alla citazione onorevole, fino a uno scorcio parallelo in terra di Francia, sono stati ripresi e approfonditi negli ultimi anni, con esiti che paiono molto circoscrivere, se non al tutto negare, la militanza del poeta sul campo più strettamente filosofico. ${ }^{47}$ E tuttavia la scelta di Marino, scelta ripetuta e mirata, di spendere la materia erotica anche in contesti ambiziosi e ufficiali, se non assume il peso di una professione filosofica, determina un'interferenza di registri e un implicito ridimensionamento dell'operazione encomiastica. Basta ricordare come era stato declinato l'omaggio per Maria de' Medici ed Enrico IV, già all'altezza delle Rime del I602, da un poeta agli esordi e dalle fortune pericolanti, ${ }^{48}$ per cogliere una poetica costante, allinsegna di oltranza e di una fiducia aggressiva nello statuto della poesia, poetica conclamata poi nelle migliaia di ottave andate a stampa nel I623.

Non sorprende dunque che, come già accadeva per altri epitalami, Venere pronuba esibisca nei suoi passaggi più accesi diversi punti di contatto con le ottave del poema. Si confronti, già nella sezione introduttiva, il corteggio degli amori con Adone XVII 70 sgg., ${ }^{49}$ e poi soprattutto una zona del lungo precetto di Venere con quanto avviene in Adone, III ISI, nel momento in cui l'Innamoramento si fa scoperto e impetuoso.

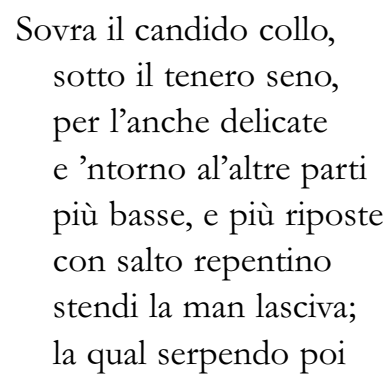


qual curiosa spia, cupida esploratrice del forte d'Onestade cerchi il più chiuso varco del bastion d'Onore e 'l più commodo sito là dove piantar possa su la rocca guardata l'amoroso vessillo. Qui tanti, e tanti aggiungi ale baciate rose accumulati baci... ${ }^{\circ}$

Con tai lusinghe il lusinghiero amante la lusinghiera dea lusinga e prega. Ella arditetta poi la man tremante gli stende al collo e dolcemente il lega. Qui, mentr'Amor superbo e trionfante l'amoroso vessillo in alto spiega, strette a groppi di braccia ambe le salme ammutiscon le lingue e parlan l'alme.

E si veda ancora l'estasi degli amanti Doria-Spinola con il punto culminante del canto dei Trastulli:

\author{
Combatti, abbatti, opprimi \\ impugna, espugna, atterra, \\ finché mancando il moto, \\ alo languir de' membri \\ rilassandosi i nodi \\ de le molli catene, \\ con flebili sussurri \\ la voce infievolisca, \\ travolti e vacillanti \\ si socchiudano i lumi, \\ stupefatte ed immote \\ agghiaccino le lingue, \\ e 'n tepide rugiade \\ sudando stilla a stilla \\ l'anima si distempri. ${ }^{5}$
}


Fa un groppo allor de l'un e l'altro core quel sommo del piacer, fin del desio. Formano i petti in estasi d'amore di profondi sospiri un mormorio. Stillansi l'alme in tepidetto umore, opprime i sensi un dilettoso oblio. Tornan fredde le lingue e smorti i volti, e vacillano i lumi al ciel travolti.

Tramortiscon di gioia ebre e languenti l'anime stanche, al ciel d'amor rapite. Gl'iterati sospiri, i rotti accenti, le dolcissime guerre e le ferite, narrar non so...

Le ottave del poema qui richiamate (come anche quelle che si è avuto occasione di citare più sopra) pertengono ai blocchi già presenti all'inizio del secolo, almeno in termini di schietta materia narrativa, e dunque in questi casi l'Adone - nel I60s poemetto per il quale Marino progettava un'uscita veneziana, al riparo da censure occhiute $-{ }^{52}$ potrebbe aver funzionato da base di partenza per nuove rielaborazioni, piuttosto che non da bacino di raccolta per versi nati entro altri progetti. Quale che sia la direzione di transito di queste tessere comuni, si profila un'omogeneità e persino un'osmosi tra registri formalmente lontani che vale a dichiarazione di una pratica poetica selezionata lucidamente, al cospetto di un pubblico allargato quanto di una compagnia scelta di committenti, e poi nobilitata attraverso la ripresa e l'implementazione di modelli classici. Era una linea annunciata, lo si è visto, in chiave difensiva nel Ragguaglio a Carlo Emanuele, e che il Marino intendeva difendere con quel discorso sullo «scrivere tenero e lascivo» che, da un certo punto in avanti, forse anche per le preoccupate ammonizioni di Guido Bentivoglio, gli sembrò difesa indispensabile per l'ingresso dell'Adone in Italia. Promesso più volte, e poi presentato come già compiuto, quel discorso rimase impigliato nelle settimane di passaggio tra Parigi e Roma, e non venne mai stampato. ${ }^{33} C^{\prime}$ è da credere che sarebbe risultato schermo troppo fragile, e che non avrebbe mutato le dinamiche prodottesi in modo repentino nei circoli romani: a seguito di una svolta rapida e profonda, la poesia mariniana, e la sua linea maestra, all'insegna di sensualità e di un classicismo senza misura, passò da prodigio di 
caposcuola a prova da spostare nell'ombra, tanto per gli ideali di profonda e disincantata autonomia che la muovevano, quanto per gli azzardi divertiti che proprio quellautonomia le suggeriva, su ogni terreno. Ed era, nelle prove raffinate di un Cesarini, nella prudenza di Bruni, e poi qualche anno dopo nella conversione letteraria di un Materdona, il segnale di chiusura di tutta una stagione.

Emilio Russo 
I. Il testo si legge in Giovan Battista Marino, Rime, a cura di M. Slawinski, 3 voll., Torino, Res, 2007, vol. I, pp. 293-96. Per una stampa perduta della canzone, antecedente alla princeps del I602, vd. G. RabONI, Geografie mariniane. Note e discussione sulle biografie seicentesche del Marino, «Rivista di letteratura italiana», IX (I99I), pp. 295-3 I I, a p. 297 n.; vd. anche E. Russo, Marino, Roma, Salerno Editrice, 2008, pp. 49-50.

2. Sulla produzione burlesca, presente e significativa già negli anni napoletani, un bilancio della bibliografia e delle notizie in Russo, Marino cit., pp. 49-57; sulla tradizione delle opere mariniane, e anche su questo versante della produzione burlesca, transitato a stampa solo dopo la morte del Marino, punto d'avvio è F. Giambonini, Bibliografia delle opere a stampa di Giambattista Marino, Firenze, Olschki, 2000.

3. In questo senso risulta efficacissimo il binomio individuato da Marzio Pieri in un articolo lontano ma ancora significativo: M. PIERI, Eros e manierismo del Marino, «Convivium», xxxvi (i 968), pp. 453-8I. Nato come recensione all'antologia curata da Asor Rosa (Giovan Battista Marino, Opere, Milano, Rizzoli, i967), il saggio approfondiva alcune delle questioni oggetto di queste pagine, liberandosi subito dalla "fama pornografica" legata al Marino, sortita in una serie di antologie mirate apparse tra fine Otto e inizio Novecento, e affrontando in particolare gli Epitalami alle pp. 464-65.

4. Per il destino complesso delle rime di Stigliani vd. il quadro ricostruito in C. Carminati, Giovan Battista Marino, l'Inquisizione, la censura, Roma-Padova, Antenore, 2008 , pp. I9 sgg. Il volume rappresenta la base essenziale per intendere pienamente l'azzardo delle scelte mariniane, sulle Rime come poi sull'Adone, alla luce delle ricadute che quelle scelte sortivano nei rapporti con la Congregazione del Sant'Uffizio.

5. È nota la pratica mariniana di distruggere gli autografi dei propri testi più azzardati: vd. la testimonianza che si legge in Giambattista Marino, Lettere, a c. di M. Guglielminetti, Torino, Einaudi, I 966, p. 71, ad Andrea Barbazza del gennaio I609: "Le cose burlesche non le mando, perché non me n'è rimaso originale, essendo state fatte solo per far favola della corte un certo buffalo che volea fare il Zanni. Ne mandai bene una copia al signor Conte Ridolfo Campeggi, dal quale potrà V.S. facilmente averle con mostrargli questa mia. Né mi curo che si trascrivano, che si veggano e che vadano in volta, poiché per queste bande non è putto che non le sappia a mente [...] Desidero solamente che l'original di mia mano s'abbrugi, per non dare adito all'altrui malignità). In questo caso il riferimento riguarda i sonetti della Murtoleide, e l'effetto di questa diffusione semiclandestina è una tradizione molto complessa, con problemi significativi di attribuzione e dunque di definizione di un corpus affidabile dei testi.

6. Si cita dal Ragguaglio a Carlo Emanuele, 34-35, sulla base dell'edizione pubblicata in E. Russo, Una nuova redazione del 'Ragguaglio a Carlo Emanuele' del Marino, «Filologia italiana», 7 (2010), pp. 107-37. Sono miei i corsivi, a testo e in nota, salvo indicazione contraria.

7. E si ricordi che il canto vIII, almeno nella sua materia narrativa, se non nell'esito concreto dei versi, probabilmente rimontava agli anni napoletani, già celebrato in 


\section{Emilio Russo}

una celebre lettera di Camillo Pellegrino del i 596. Sul rilievo di questa diacronia interna al poema vd. quanto detto oltre, in relazione ad altri testi mariniani. Per il poema si fa riferimento all'edizione Giovan Battista Marino, Adone, a c. di E. Russo, 2 voll., Milano, Rizzoli, 2013.

8. Per la ricostruzione del destino editoriale di queste ottave, cadute per prudenza in alcune ristampe successive, vd. E. Russo, Studi su Tasso e Marino, Roma-Padova, Antenore, 2005, pp. IOI-I09.

9. Vd. la testimonianza che si legge in Lettere a diversi del Signor Girolamo Magagnati, a c. di L. Salvetti Firpo, con premessa la vita di Girolamo Magagnati, a c. di C. Carabba e G. Gasparri, Firenze, Olschki, 2006, pp. 25-26, lettera num. 37.

ı. Vd. E. Torchio, Marino amante ovidiano, «Studi secenteschi», XLI (2000), pp. 89-I 2 I.

i i. Al riguardo, per tutta questa sezione, vd. Carminati, Giovan Battista Marino, l'Inquisizione e la censura cit., pp. 73-91, e in particolare pp. 86-87.

I 2. Una rassegna dei precedenti classici, ancora valida seppure lontana nel tempo, si legge in C. Moreldi, L'epitalamio nella tarda poesia latina, «Studi di filologia classica», XVIII (I910), pp. 319-432, con particolare riguardo, alle pp. 337 sgg., a Claudiano e ai suoi Fescennina, modelli tra quelli più vicini all'ispirazione mariniana. Al riguardo si ricordi G. F. Damiani, Sopra la poesia del cavalier Marino, Torino, Clausen, I893, pp. Io0 sgg., con una serie di annotazioni relative agli Epitalami.

I3. Per un repertorio delle pubblicazioni per nozze, su spettro cronologico ampio, vd. O. PINTO, Nuptialia, Saggio di bibliografia di scritti italiani pubblicati per nozze dal I484 al 1799, Firenze, Olschki, I97 I. Più mirato sulla zona di pertinenza mariniana, seppure su un caso specifico, vd. il saggio di D. Boillet, Marino, Rinaldi, Achillini, Campeggi, Capponi e altri in una raccolta bolognese per nozze (I607), «Studi secenteschi», XLV (20I4), i.c.s., con diffusa argomentazione sul contesto bolognese e sulle relazioni reciproche tra i testi della raccolta.

14. Lettera Claretti, 39 (secondo l'edizione presente in Russo, Studi su Tasso e Marino cit., pp. I 54-55).

I 5. Sulla Francia consolata si ricordi l'edizione e le annotazioni che l'accompagnano pubblicate in M. Pieri, Per Marino, Padova, Liviana, i 976, pp. 35 6-88.

I6. Raccolgo qui alcuni degli studi disponibili sugli epitalami mariniani, a partire dal lontano A. Borzelui, Gli epitalami del Marino e particolarmente del terzo, Napoli, Marzano, i924. Vd. poi l'importante saggio di M. Dell'Ambrogio, Tradurre, imitare, rubare: appunti sugli 'Epitalami' del Marino, in Forme e vicende. Per Giovanni Pozzi, a c. di O. Besomi, G. Gianella, A. Martini, G. Pedrojetta, Padova, Antenore, I 988, pp. 269-93. Mirato soprattutto agli equilibri francesi il saggio di D. Boillet, Marino et le "Afuctuations de la France »: il 'Tempio' (I6IS) et les 'Epitalami' (I6I6), in $L^{\prime}$ actualité et sa mise en écriture dans l'Italie de $X V^{e}-X V I I^{e}$ siècles. Actes du Colloque International de Paris, 21-22 octobre 2002, réunis et présentés par D. Boillet et C. Lucas, Paris, Cirri, 2004, pp. 205-43. Vd. adesso D. Bolllet, Les 'Epitalami' de Giovan Battista Marino: le livre et sa fabrique, in Forme e occasioni dell'encomio tra Cinque- 
cento e Seicento/Formes et occasions de la louange entre $X V T^{e}$ et $X V I I^{e}$ siècle, a c. di D. Boillet e L. Grassi, Lucca, Pacini Fazzi, 20 I , pp. i8 I-2 I 2; S. Morando, Quando il potente ama. Raffigurazioni encomiastiche negli 'Epithalami' (I6I6) di Giovan Battista Marino, in Le maschere del potere. Leadership e culto della personalità nelle relazioni fra gli stati dall'antichità al mondo contemporaneo, a c. di L. Santi Amantini e F. Gazzano, Roma, L'Erma di Bretschneider, 201 3, pp. I 5 3-76.

17. Vd. quanto si legge in Marino, Lettere, cit., p. 35. Per gli Epitalami occorre ricordare l'edizione dei testi che venne approntata da Michele Dell'Ambrogio in una tesi discussa presso l'università di Friburgo nel 1973 sotto la guida di padre Giovanni Pozzi; la tesi è attualmente consultabile presso la Biblioteca dell'Istituto di Italiano dell'ateneo svizzero. Un primo quadro sulla tradizione a stampa dei testi in Russo, Marino cit., pp. I 58-59. Una nuova edizione critica e commentata degli Epitalami è ora prevista per le cure di Carlo Alberto Girotto e di Paolo Marini.

I8. «Mentre i duo casti amanti, / Aminta ed Amarilli / fean de le nozze lor superbo il Tebro»; si cita da qui in avanti dalla princeps parigina del I6 6 (qui Marino, Epitalami, p. I69), con semplice rimando al numero di pagina. Minimi gli interventi qui operati nella trascrizione dei testi: distinzione $\mathrm{u} / \mathrm{v}$, abbassamento ove necessario delle maiuscole sempre presenti a inizio verso nella princeps, eliminazione delle $h$ etimologiche o pseudo-etimologiche, resa di accenti e apostrofi secondo l'uso moderno.

I9. Marino, Epitalami, p. I69: «Fileno acceso ed ebro / d'alto furor, Filen che 'n riva al'acque / dele Sirene nacque». Il pescator Fileno è limpida controfigura del Marino, qui, all'altezza del I602, in una delle primissime occorrenze, se si ricorda che il precedente nome pastorale impiegato dal Marino, Carino, aveva caratterizzato alcune prove della prima stagione romana.

20. Marino, Epitalami, p. I69: «O Notte, o del'antico / Erebo tenebroso / tacita figlia, e del'Oblio profondo; / o del Silentio amico, / del Sonno, e del Riposo / placida madre, ond'ha ristoro il mondo». Si ricordi anzi tutto il precedente di Della Casa, Rime, LIV, I-4, opportunamente ridisegnato ( $(\mathrm{O}$ sonno, o de la queta, umida, ombrosa / notte placido figlio; o de' mortali / egri conforto, oblio dolce de' mali / sì gravi ond'è la vita aspra e noiosa»), nonché i celebri precedenti virgiliani e ovidiani. In ambito mariniano, più che Adone, XIII 58 , sarà da ricordare un passaggio delle Rime del I602 (Rime lugubri, 7: «Era la notte, e tenebrosa e nera / ombra mortal da l'Erebo traea, / e, quasi pompa funerale, avea / l'aere coperto, e la stellata spera»).

2i. Marino, Epitalami, pp. i70-71. L'invocazione finale del brano è largamente topica (vd. già Catullo, Carmina, LXI e LXII), e scandisce ad esempio la zona conclusiva del Pastor fido (atto v, scena 9), ma si legge anche in TAsso, Rime, 538 , nell'epitalamio composto dal Tasso per le nozze di Francesco Maria della Rovere e di Lucrezia d'Este, o ancora come refrain martellante in altro testo tassiano per nozze (Rime, i 366). Si ricordi, retrostante, l'epitalamio di Bernardo Tasso per le nozze del duca di Mantova, probabilmente noto al Marino (si legge in BerNARDo TAsso, Rime, Torino, Res, I 995, II Iо I, vol. I pp. 24I-48).

22. Marino, Epitalami, p. I 7 I. 


\section{Emilio Russo}

\section{Marino, Epitalami, p. 172.}

24. Elementi analoghi, ad esempio, ancora in TAsso, Rime, 538 ( $«$ Solvi, o felice sposo, il casto cinto / che severo custode a te riserba / puri e 'n cielo graditi almi diletti: / vivi, or che puoi, tra que' bei nodi avvinto, / ché Marte omai questa tua etade acerba / par ch'a le dure sue fatiche affretti.»). Qui però conviene soprattutto ricordare Rime, 569, epitalamio scritto da Tasso nel i 568 per le nozze tra Marfisa d'Este e don Alfonso d'Este, che offre toni di sensualità aperta, assai distante dalle numerose altre declinazioni tassiane del genere (vd. almeno Rime, 887, I 25 I, I 363, I457). Così uno scorcio del testo del I 578: «Questa bella guerriera / che, o contra Amor s'accinga / o per lui cinga l'arme, è vincitrice, / da l'amorosa schiera / lunge se 'n va solinga / e scompagnata in guisa di fenice / [...] / ecco, punta è da l'armi / quasi cervetta al varco / e tutta arde d'amor la verginella; / ma pur talor rubella / si mostra nel sembiante, / e vaga e ritrosetta / minaccia e 'nsieme alletta, / or di guerriera in atto ed or d'amante; / e'n un dubbia e confusa, / fra vergogna e desir, brama e ricusa. // Va fra gli sdegni ed osa, / regio garzon, ch'al fine / pietosa fia questa beltà crudele! / Si coglie intatta rosa / fra le pungenti spine, / e fra gli aghi de l'api il dolce mele. / Lascia pur ch'ella cele / sue voglie e ti contrasti; / rapisci: più graditi / sono i baci rapiti / e più soavi son quanto più casti; / non cessar fin che 'l sangue / non versa e vinta a te sospira e langue. // Sacra lieto trofeo / del bel cinto disciolto / e de le spoglie sue di sangue sparte, / e i giochi d'Imeneo / rinnova in nodi accolto / più bei di quei ch'unir Ciprigna a Marte» (TASsO, Rime, 569, vv. 40 sgg.).

25. Vd. ad esempio Adone, III I 5 I, citato distesamente più avanti.

\section{Marino, Epitalami, p. 173.}

27. Il testo si legge in Marino, Epitalami, p. i77. Al riguardo vd. A. Colombo, Versi autografi del Marino per Ranuccio Farnese e Margherita Aldobrandini, «Lettere italiane» XLIII (I991), pp. 374-90.

28. Vd. G. Pozzi, Guida alla lettura, in Giovan Battista Marino, Adone, a c. di G. Pozzi, 2 voll., Milano, Mondadori, i976, vol. II, pp. 403-404.

29. Dell'Ambrogio, Tradurre, imitare, rubare cit., pp. 279-80.

30. Marino, Epitalami, pp. 57-92. Nell'ambito del discorso qui condotto, e soprattutto in relazione alla sezione conclusiva di questa pagine, andrà ricordata la prossimità della descrizione di Amore infido e irrefrenabile entro Il balletto delle Muse (ed. cit., pp. 6o-6r) e quella che si legge nel canto vi dell' Adone (vi i 6 I sgg.); e simmetricamente quella di Amore come passione divina che si legge sempre ne Il balletto delle Muse, ed. cit., pp. 61-62 («Dispensiero cortese / di leggittime gioie, / imperador de' nobili desiri / illustrator de' torbidi pensieri, / regolator degli sfrenati affetti, / temperator dei malaccesi ardori, / generosa virtù, puro desio / del simile, e del bello / dolce innesto de' corpi / santa pace de' cori...»), e poi in Adone, I I I 6: «Tu virtù somma de' superni giri, / dispensier de le gioie e de' piaceri, / imperador de' nobili desiri / illustrator de' torbidi pensieri, / dolce requie de' pianti e de' sospiri, / dolce union de' cori e de' voleri, / da cui Natura trae gli ordini suoi / dio de le meraviglie e che non puoi?». 
3i. Marino, Epitalami, p. I48; l'oriche per errore nella princeps parigina.

32. MARINo, Epitalami, pp. I49-50.

33. Vd. anche Adone, iv 33 («Allor mi stringe entro le braccia e mille / groppi mi porge d'infocati baci, / poi per l'oro immortal, per le faville / de le quadrella mie, de le mie faci, / quanto può mi scongiura»). Si ricordi tuttavia una tessera delle rime del $\mathrm{I}_{602}$, un sonetto delle Rime boscherecce, presentato come esplicita riscrittura di Catullo, ove ricorrono le medesime immagini: «Baciami, bacia e dammi, o cara Fille, / e mille e mille baci e cento e cento, / poi cento et altri cento et altri mille; / ch'altro piacer che te baciar non sento. / [...] / Tessiam groppi di baci e di sospiri, / e fra le perle e fra' rubin mordaci / l'umidetta talor serpa e s'aggiri. / E se 'l baciar ti stanca, arresta i baci, / pur che la tua ne la mia bocca spiri / l'odorate, ond'io vivo, aure vivaci»; si cita da Giovan Battista Marino, Rime boscherecce, a c. di J. Hauser-Jakubowicz, Ferrara-Modena, Istituto di Studi Rinascimentali-Panini, I99I, num. 38, p. 64.

34. Marino, Epitalami, p. I 5 2: «Or in sì fatto agone, / mentre a strette contese / di ripari, e d'offese / son la bella Guerriera, e 'l bel Campione / su 'l fin dela tenzone / l'armi drizzando al'ultime percosse / con incontro d'Amore / l'una è colta nel sen, l'altro nel core. // Così vinto l'invitto / mentre trafige, e 'mpiaga / resta in battaglia il piagator trafitto».

35. Al riguardo vd. le considerazioni di Pozzi, Guida alla lettura cit., vol. II, p. 404.

36. Marino, Epitalami, pp. i 2-53.

37. Il testo mariniano non venne compreso nella raccolta in onore degli sposi, andata a stampa nel i6o9 (Nelle nozze del co. Ercole Pepoli et D. Vittoria Cibò, Bologna, eredi di Giovanni Rossi, I 609); per le dinamiche relative all'edizione vd. Boillet, Marino, Rinaldi, Achillini, Campeggi, Capponi e altri in una raccolta bolognese per nozze (I607) cit.; inoltre Marino, Lettere, cit., p. 70 num. 45.

38. Marino, Epitalami, pp. i 56 , i 59 .

39. Per i dettagli su questo progetto vd. Russo, Marino cit., pp. 239-45, con la bibliografia ivi richiamata.

40. Vd. Dell'Ambrogio, Tradurre, imitare, rubare: appunti sugli 'Epitalami' del Marino cit., pp. 280-8I.

4I. Vd. sopra nota I4.

42. Vd. lo specchietto riassuntivo in Carminati, Giovan Battista Marino, l'Inquisizione, la censura cit., p. 385 .

43. Vd. il quadro ricostruito in V. Farina, Giovan Carlo Doria promotore delle arti a Genova nel primo Seicento, Firenze, Edifir, 2002.

44. Accanto all'epitalamio di Claudiano per Palladio e Celerina, la cui ripresa è stata ricordata in Dell'Ambrogio, Tradurre, imitare, rubare: appunti sugli 'Epitalami' del Marino cit., pp. 276-77, possibile che il Marino abbia anche ripreso l'epitalamio di Stella e Violentilla di STAZio, Silvae, i 2. 


\section{Emilio Russo}

45. Marino, Epitalami, p. io4.

46. BorzelLi, Gli epitalami del cavalier Marino cit., pp. i7-2 I, con rinvio anche agli ambienti di formazione del Marino, alla Napoli dell'Accademia degli Svegliati, al circolo di Cortese e ai possibili influssi telesiani.

47. Vd. F. P. Raimondi, Tracce vaniniane nell' 'Adone' del Marino?, in Marino e il Barocco, da Napoli a Parigi, a c. di E. Russo, Alessandria, Edizioni dell'Orso, 2009, pp. 347-83, con accurata discussione della bibliografia precedente, a partire da un paio di saggi importanti di Giorgio Fulco.

48. Vd. Rime eroiche, 6 (in Marino, Rime, ed. cit., vol. I); inoltre Russo, Marino cit., p. 90.

49. In relazione a MARINo, Epitalami, pp. IO2-103.

50. Marino, Epitalami, pp. i io-i i.

5. Marino, Epitalami, p. i i 4 .

52. Vd. Marino, Lettere cit., p. 52, in una lettera dell'aprile i605 a Bernardo Castello.

53. Sul progetto di questo discorso vd. Russo, Marino cit., pp. 337-39, e rimandi relativi. 